\title{
Empirical Foundation of Space and Time
}

\author{
László E. Szabó \\ Department of Logic, Institute of Philosophy \\ Eötvös University, Budapest
}

\begin{abstract}
I will sketch a possible way of empirical/operational definition of space and time tags of physical events, without logical or operational circularities and with a minimal number of conventional elements. As it turns out, the task is not trivial; and the analysis of the problem leads to a few surprising conclusions.
\end{abstract}

\section{Introduction}

The central issue of special relativity is the comparison of space and time tags of physical events, defined in different inertial frames of reference. However, the question of how these space and time tags are defined in one single frame of reference is considered as unproblematic and is usually neglected. In this paper, I will focus on this second question.

When I say "definition", I mean empirical definition, somewhat similar to Reichenbach's "coordinative definitions", Carnap's "rules of correspondence", or Bridgman's "operational definitions"; which give an empirical interpretation of the theory.

Einstein, at least in his early writings, strongly emphasizes that all spatio-temporal terms he uses are based on operations applying measuring rods, clocks and light signals. In his 1905 paper, he describes the measurement of the length of a rod in an arbitrary (moving) inertial frame of reference as follows:

The observer moves together with the given measuring-rod and the rod to be measured, and measures the length of the rod directly by superposing the measuring-rod, in just the same way as if all three were at rest.

And this is a typical description of the empirical meaning of length or distance. However, these usual operational definitions so often suggested in the textbook literature 
are untenable; they are full of obvious circularities. It is not my aim here to address the problems in question, because the upshot of these considerations is also quite common in the more sophisticated part of the literature of space-time physics: In order to avoid these obvious circularities and to minimize the conventional elements in the empirical foundation of our physical theory of space and time, we must avoid using standard measuring rod in the definition of distance and using slow transportation of the standard clock in the definition of time tags, and the likes. We must also abstain from relying on the concept of rigid body, reference frame, and inertial motion. Instead, we have to use one standard clock and light signals.

Of course, using one standard clock and light signals for coordination of spacetime is an old idea; as old as the widespread belief that the task is as trivial as it seems from the two-dimensional textbook examples, and that the resulted spatio-temporal structure is, at least locally, necessarily identical with the standard space-time geometry of special relativity. What will be new in our analysis is the consequent performance of this task without operational circularities. As we will see, the task is not trivial; and the analysis of the spatio-temporal conceptions so obtained will raise some still open-although experimentally testable-questions.

\section{Empirical definition of space and time tags}

First we chose an etalon clock. That is to say, we chose a system (a sequence of phenomena) floating somewhere in the universe. Without loss of generality we may stipulate that this is an equipment having a pointer and the readings are real numbers. There is no assumption that this is a clock measuring "proper time". There is no assumption that it "runs uniformly". And there is no assumption that it is "at rest" relative to anything, or that it is of "inertial motion". The reason is that none of these concepts is defined yet.

We will call "marker" an equipment which can be triggered by a physical event and can transmit and receive modulated radio waves containing some information. Assume we have as many markers as we need, with the following functions:

1. There is a distinguished marker floating together with the standard clock and continuously transmitting the actual reading of the standard clock.

2. The others continuously receive the regular time signals from the standard clock.

3. They can transmit radio signals containing the following information: a) an ID code of the device and information about the standard clock reading, so from the signal they send it always can be known which device was the transmitter and what was the standard clock reading received by the transmitter at the moment of the emission of the signal, b) information about the event on the occasion of which the signal was transmitted.

4. They can receive the signals transmitted by the others.

By the emission of a radio signal the marker marks an event. It is far from obvious, however, what must be regarded as an event in general-prior to the concepts of time 


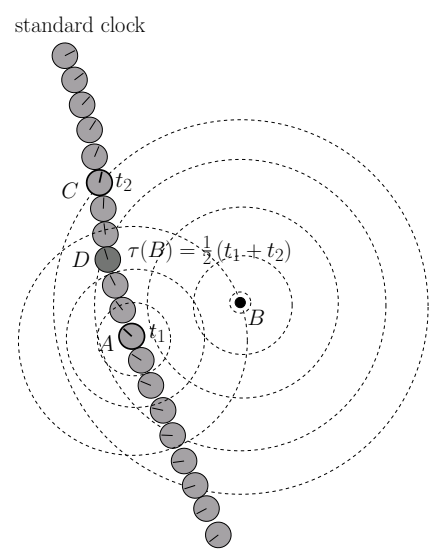

Figure 1: Operational definition of time tags. (This is just a symbolic sketch, not a real "two dimensional space-time diagram" or the like.)

and distance. (See Brown 2005, pp. 11-14.) We do not dwell on this problem here. The reader can easily imagine various operational solutions of how to use a marker for marking various physical events/phenomena.

\subsection{Time}

Consider the experimental arrangement in Fig. 1. The marker at the standard clock emits a radio signal at clock-reading $t_{1}$ (event $A$ ). The signal is received by another marker which immediately emits another signal (event $B$ ). This "reflected" signal is detected by the marker at the standard clock at $t_{2}$ (event $C$ ). We assume, as an empirical fact, that the clock we have chosen is such that a given reflected signal is received by the standard clock only once, at reading $t_{2}$, and

$$
t_{2} \geq t_{1}
$$

by which we have chosen, conventionally, an "arrow of time" (not the arrow of physical processes in time; see Price 1996, p. 16 and 58). (In fact, we made two choices here. One is the choice of the direction of the parametrization of the clock's pointer positions (1). There is however a more important one: by applying the terms "sending" and "receiving" a signal, we previously determined the causal order of events $A$ and $C$. To what extent this causal order is purely conventional? How can we-without prior spatio-temporal conceptions - distinguish whether an event is a "sending" or a "receiving" of a signal? How is this choice of causal order related to the change of information content of the signal? To what extent this choice is determined by our free will and free action experience at the modulation of the radio waves? Is this freedom an objective openness of future or merely a subjective experience? These are delicate metaphysical questions into the discussion of which it is not our present purpose to enter.) 
Definition (A1) The absolute time tag of event $B$ is the following:

$$
\tau(B):=t_{1}+\varepsilon\left(t_{2}-t_{1}\right)
$$

where $\varepsilon=\frac{1}{2}$ by convention. (Of course, it could be a contingent fact of nature that $t_{2}=t_{1}$, in which case the choice of the value of $\varepsilon$ would not matter.)

It is important to emphasize that the choice of using radio signals in definition (A1) is purely conventional. This choice is by no means justified by the "constancy and isotropy of the (round-trip) velocity of light"; simply because we are prior to any spatio-temporal concepts that would make any statement about the "velocity" of light meaningful.

\subsection{Distance and the problem of "rest"}

Denote $S_{\tau}$ the set of simultaneous events with time tag $\tau$. One might think that we are ready to define the spatial distance between two points of space, that is distance between two simultaneous events. Surely, we can define the distance between the simultaneous events $D$ and $B$ in Fig. 1 as $\frac{1}{2}\left(t_{2}-t_{1}\right) c$, where the value of $c$ is taken as a convention. In this way however, as a little reflection shows, we can define the distance only from the standard clock, but there is no way to extend this definition for arbitrary pair of simultaneous events. In order to define the distance between two arbitrary simultaneous events we need further preparations.

We would like to base the definition of distance to the definition of time: the distance between two points in a given $S_{\tau}$ will be defined through the period of time in which a radio signal runs "from the one point to the other". Therefore, instead of signals sent and received by the marker at the standard clock, we will use radio signals "sent from the one point and received at the other". However, we encounter the following difficulty. We would like to define distance between simultaneous events; but the travel of the signal takes some time; the emission of the signal and the receiving of the signal are not simultaneous events. Whose distance is the one measured by the time of travel of the signal - and when? The distance obtained by means of the time of travel of the signal depends on the concept of "rest"; the concept of "being at the same place at different times" (Fig 2). So, in order to define the distance of simultaneous events we need a previous concept of "rest"; and, moreover, we have to define this concept by the only means of the standard clock and radio signals.

It is necessary to be careful of a possible misunderstanding. Although they are close to each other, the problem we are addressing here is different from the problem of persistence of physical objects (Butterfield 2005). What we would like to define is the identity of two locuses of space at two different times, and not the genidentity of the physical objects occupying these locuses. One might think that some definition of genidentity of physical objects must be prior to our operational definition of space and time tags, at least in the case of the standard clock. This is, however, not necessarily the case. The standard clock is just an ordered (ordered by the clock readings) sequence of physical events, but without the further metaphysical assumption that these events belong to the same physical object. (We definitely do not make such assumption in the case of a "clock-like" sequence of events that we will call a time sequence below.) 


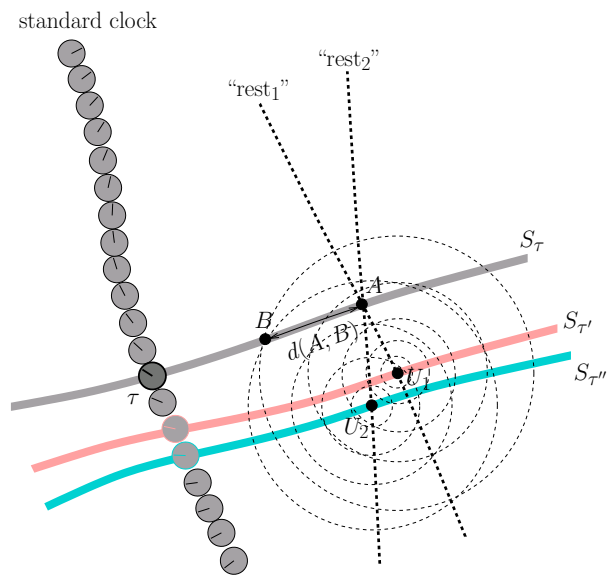

Figure 2: The distance defined by means of the time of travel of the radio signal depends on the concept of "rest"; the concept of "being at the same place at different times". In general, $\tau(B)-\tau\left(U_{1}\right) \neq \tau(B)-\tau\left(U_{2}\right)$

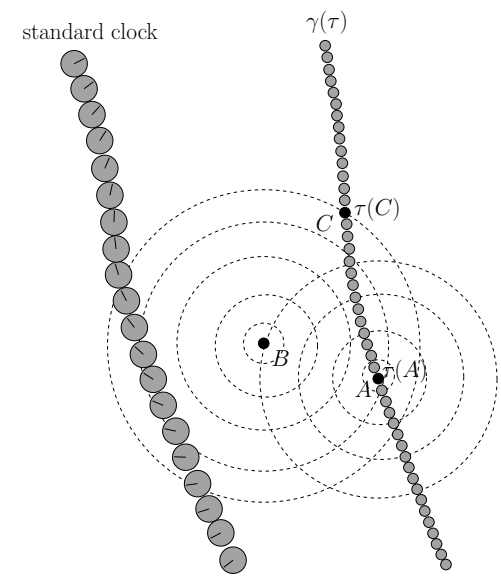

Figure 3: Clock-like time sequence

Definition (A2) A one-parameter family of events $\gamma(\tau)$ is called time sequence if $\gamma(\tau) \in S_{\tau}$ for all $\tau$.

One has to recognize that a time sequence is a "clock-like" sequence of events. For every event, one can define a time-like tag in the same way as (A1): Event $A$ (Fig. 3) is marked with the emission of a radio signal at time $\tau(A)$. The signal is reflected at event $B$. Event $C$ is the first detection of the reflected signal at time $\tau(C)$. We define the following time-like tag for event $B$ :

$$
\tau^{\gamma}(B):=\tau(A)+\varepsilon(\tau(C)-\tau(A))
$$




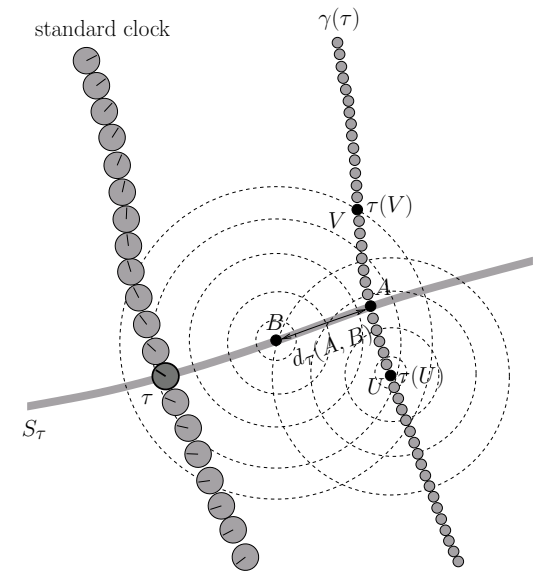

Figure 4: The distance between two simultaneous events

(If there is no detection of the reflected signal at all, then, say, $\tau^{\gamma}(B):=\infty$.)

It is an empirical fact that $\tau^{\gamma}(B) \neq \tau(B)$ in general. It is another empirical observation however that for some particular cases $\tau^{\gamma}(B)=\tau(B)$.

Definition (A3) A time sequence $\gamma(\tau)$ is a rest time sequence if for every event $B$ $\tau^{\gamma}(B)=\tau(B)$.

Whether or not there exist rest time sequences is an empirical question. We stipulate the following:

Empirical fact (E1) For any event $A$ there exists a unique rest time sequence $\gamma(\tau)$ such that $A=\gamma(\tau(A))$.

Rest time sequence is a concept defined only by means of the standard clock and radio signals. It singles out a "world line" through every event, that will play the role of the "world line of a particle being at rest relative to the standard clock".

Now we are ready to define the distance between simultaneous events.

Definition (A4) The absolute distance between two simultaneous evens $A, B \in S_{\tau}$ is operationally defined in the following way. Take a rest time sequence $\gamma$ such that $A=\gamma(\tau)$ (Fig. 4). Let $U=\gamma(\tau(U))$ be an event marked with the emission of a radio signal at absolute time $\tau(U)$, such that the signal is received and reflected at event $B$. The detection of the reflected signal marks the event $V=\gamma(\tau(V))$ of time tag $\tau(V)$. The absolute distance is

$$
d_{\tau}(A, B):=\frac{1}{2}(\tau(V)-\tau(U)) c
$$

where $c=299792458 \frac{m}{s}$ by convention. 
We know from (1) that for all $A, B \in S_{\tau}$

$$
\begin{aligned}
& d_{\tau}(A, B) \geq 0 \\
& d_{\tau}(A, A)=0
\end{aligned}
$$

However, the following facts cannot be known without further empirical observations:

Empirical fact (E2) For all $A, B, C \in S_{\tau}$

$$
\begin{aligned}
d_{\tau}(A, B) & =0 \text { only if } A=B \\
d_{\tau}(A, B)+d_{\tau}(B, C) & \geq d_{\tau}(A, C) \\
d_{\tau}(A, B) & =d_{\tau}(B, A)
\end{aligned}
$$

The following proposition is however derivable:

Lemma 1 Let $\gamma_{1}$ and $\gamma_{2}$ be arbitrary two rest time sequences. For any two moments of absolute time $\tau$ and $\tau^{\prime}$

$$
d_{\tau}\left(\gamma_{1}(\tau), \gamma_{2}(\tau)\right)=d_{\tau^{\prime}}\left(\gamma_{1}\left(\tau^{\prime}\right), \gamma_{2}\left(\tau^{\prime}\right)\right)
$$

Having distance defined on a given $S_{\tau}$, we introduce the following abbreviations:

$$
\begin{aligned}
\operatorname{Cong}_{\tau}(A, B, C, D) & \Longleftrightarrow d_{\tau}(A, B)=d_{\tau}(C, D) \\
\operatorname{Bet}_{\tau}(A, B, C) & \Longleftrightarrow d_{\tau}(A, C)=d_{\tau}(A, B)+d_{\tau}(B, C)
\end{aligned}
$$

In terms of these abbreviations we formulate the following - not necessarily newempirical facts:

(E3) $\forall A \forall B$ Cong $_{\tau}(A, B, B, A)$

(E4) $\forall A \forall B \forall C \operatorname{Cong}_{\tau}(A, B, C, C) \rightarrow A=B$

(E5) $\quad \forall A \forall B \forall C \forall D \forall E \forall F$ Cong $_{\tau}(A, B, C, D)$

$\wedge \operatorname{Cong}_{\tau}(C, D, E, F) \rightarrow \operatorname{Cong}_{\tau}(A, B, E, F)$

(E6) $\forall A \forall B \operatorname{Bet}_{\tau}(A, B, A) \rightarrow A=B$

(E7) $\left.\forall A \forall B \forall C \forall D \forall E \operatorname{Bet}_{\tau}(A, D, C) \wedge \operatorname{Bet}_{\tau}(B, E, C)\right)$

$\rightarrow \exists F\left(\operatorname{Bet}_{\tau}(D, F, B) \wedge \operatorname{Bet}_{\tau}(E, F, A)\right.$

(E8) $\exists E \forall A \forall B A \in \alpha \wedge B \in \beta \rightarrow \operatorname{Bet}_{\tau}(E, A, B)$

$\rightarrow \exists F \forall A \forall B A \in \alpha \wedge B \in \beta \rightarrow \operatorname{Bet}_{\tau}(A, F, B)$

where $\alpha$ and $\beta$ are two sets of events in $S_{\tau}$.

(E9) $\exists A \exists B \exists C \exists D \exists E \neg D=E \wedge$ Cong $_{\tau}(A, D, A, E)$

$\wedge \operatorname{Cong}_{\tau}(B, D, B, E) \wedge \operatorname{Cong}_{\tau}(C, D, C, E)$

$\wedge \neg \operatorname{Bet}_{\tau}(A, B, C) \wedge \neg B e t_{\tau}(B, C, A) \wedge \neg B e t_{\tau}(C, A, B)$ 


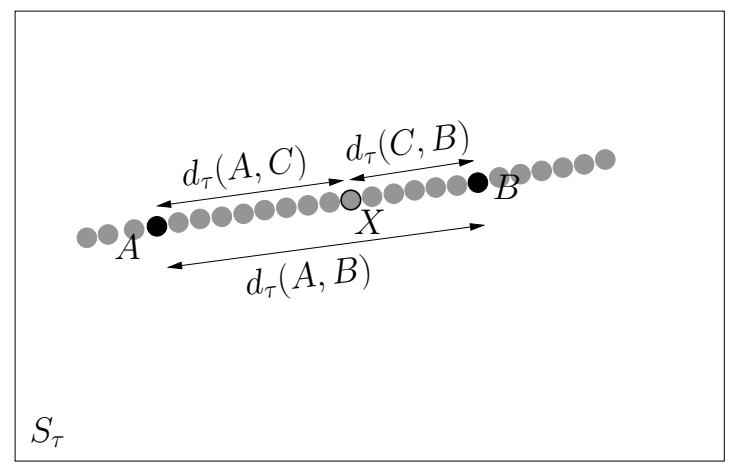

Figure 5: Straight line

(E10) $\forall A \forall B \forall C \forall D \forall E \forall F \neg D=E \wedge \neg D=F \wedge \neg E=F$

$\wedge \operatorname{Cong}_{\tau}(A, D, A, E) \wedge \operatorname{Cong}_{\tau}(A, D, A, F)$

$\wedge \operatorname{Cong}_{\tau}(B, D, B, E) \wedge \operatorname{Cong}_{\tau}(B, D, B, F)$

$\wedge \operatorname{Cong}_{\tau}(C, D, C, E) \wedge \operatorname{Cong}_{\tau}(C, D, C, F)$

$\rightarrow \operatorname{Bet}_{\tau}(A, B, C) \vee B t_{\tau}(B, C, A) \vee B_{e} t_{\tau}(C, A, B)$

(E11) $\forall A \forall B \forall C \forall D \forall E \forall F \operatorname{Bet}_{\tau}(A, B, F) \wedge \operatorname{Cong}_{\tau}(A, B, B, F)$

$\wedge \operatorname{Bet}_{\tau}(A, D, E) \wedge \operatorname{Cong}_{\tau}(A, D, D, E)$

$\wedge B_{\tau} t_{\tau}(B, D, C) \wedge \operatorname{Cong}_{\tau}(B, D, D, C)$

$\rightarrow$ Cong $_{\tau}(B, C, F, E)$

(E12) $\forall A \forall B \forall C \forall D \forall E \forall F \forall G \forall H \neg A=B \wedge B e t_{\tau}(A, B, C)$

$\wedge \operatorname{Bet}_{\tau}(E, F, G) \wedge \operatorname{Cong}_{\tau}(A, B, E, F)$

$\wedge \operatorname{Cong}_{\tau}(B, C, F, G) \wedge \operatorname{Cong}_{\tau}(A, D, E, H)$

$\wedge \operatorname{Cong}_{\tau}(B, D, F, H) \rightarrow \operatorname{Cong}_{\tau}(C, D, G, H)$

(E13) $\forall A \forall B \forall C \forall D \exists E \operatorname{Bet}_{\tau}(D, A, E) \wedge \operatorname{Cong}_{\tau}(A, E, B, C)$

The quantification runs over $S_{\tau}$. In brief, we stipulate, as an empirical fact, that the two relations $\operatorname{Cong}_{\tau}$ and Bet $_{\tau}$, determined by the distances of simultaneous events, satisfy the axioms of 3-dimensional Euclidean geometry, namely Tarski's axioms of 3-dimensional Euclidean geometry (Tarski and Givant 1999). It must be emphasized that all the statements (E3)-(E13) are stipulated, via inductive generalization, merely on the basis of observations about distances of simultaneous events.

\subsection{Spatial coordination}

Within this axiomatic framework, one can define the basic geometrical concepts in the usual way; and one can derive a body of theorems, well known from the textbooks on Euclidean geometry. Below are a few of the typical definitions and theorems we will use in the construction of space tags. 


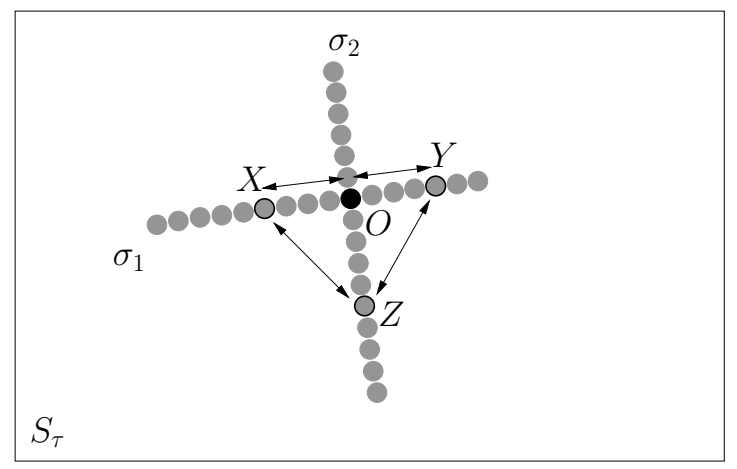

Figure 6: Orthogonal lines

Definition $A$ subset $\sigma \subset S_{\tau}$ is called (straight) line if satisfies the following conditions (Fig. 5):

1. for any $A, B, C \in \sigma$ exactly one of the following three relations hold:

$$
\begin{aligned}
& d_{\tau}(A, C)+d_{\tau}(C, B)=d_{\tau}(A, B) \\
& d_{\tau}(A, B)+d_{\tau}(B, C)=d_{\tau}(A, C) \\
& d_{\tau}(B, A)+d_{\tau}(A, C)=d_{\tau}(B, C)
\end{aligned}
$$

2. $\sigma$ is maximal for property 1 .

Definition Let $\sigma_{1}$ and $\sigma_{2}$ be two lines in $S_{\tau}$ such that $\sigma_{1} \cap \sigma_{2}=\{O\}$ (Fig. 6). $\sigma_{2}$ is orthogonal to $\sigma_{1}$ if for every $Z \in \sigma_{2}$ and for every $X, Y \in \sigma_{1}$

$$
d_{\tau}(X, O)=d_{\tau}(O, Y) \Leftrightarrow d_{\tau}(X, Z)=d_{\tau}(Y, Z)
$$

Theorem For every $A, B \in S_{\tau}$ there exists a unique line containing $A$ and $B$.

Theorem Let $A \in S_{\tau}$ be an arbitrary event and let $\sigma_{1} \subset S_{\tau}$ be an arbitrary line. There always exists a line $\sigma_{2}$ orthogonal to $\sigma_{1}$, such that $A \in \sigma_{2}$.

Definition Using the notations of the above theorem, let $\sigma_{1} \cap \sigma_{2}=\{O\}$. Event $O$ is called the orthogonal projection of A to $\sigma_{1}$. Distance $d_{\tau}(A, O)$ is called the distance of A from $\sigma_{1}$.

Definition Let $\sigma_{1} \subset S_{\tau}$ be a line. A line $\sigma_{2}$ is parallel to $\sigma_{1}$ if for all $X \in \sigma_{2}$ the distance of $X$ from $\sigma_{1}$ is the same.

Theorem Let $\sigma_{1} \subset S_{\tau}$ be a line and let $C \in S_{\tau}$ be an arbitrary event. There exists exactly one line $\sigma_{2}$ such that $C \in \sigma_{2}$ and $\sigma_{2}$ is parallel to $\sigma_{1}$. 


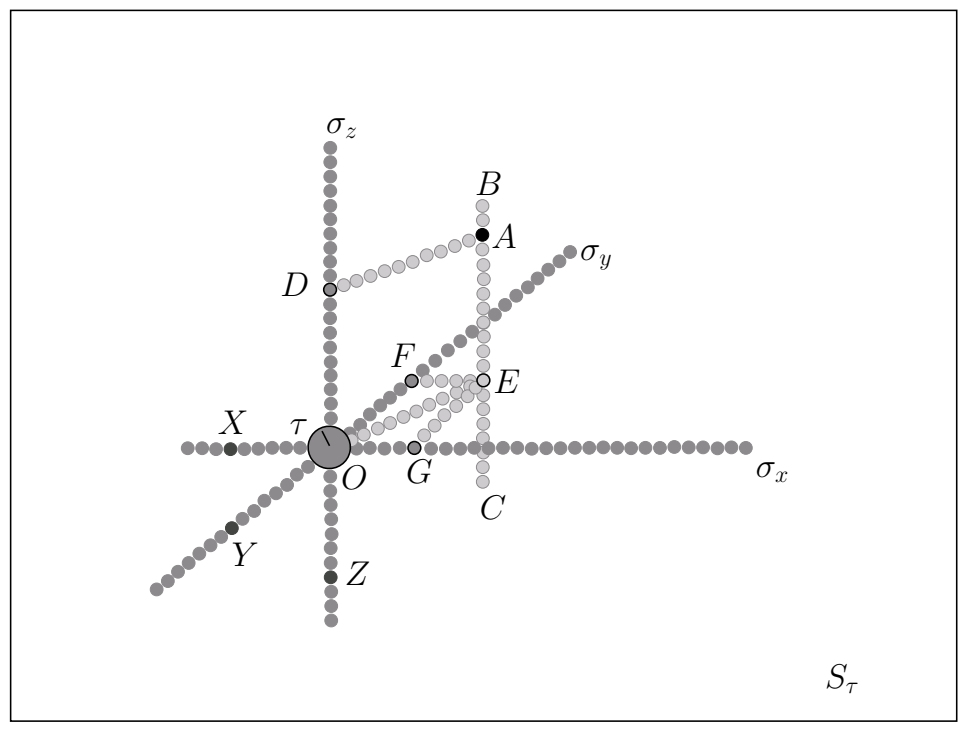

Figure 7: Cartesian coordinates in $S_{\tau}$

Definition Let $A, B \in \sigma$ be two events on line $\sigma$. Line segment between events $A, B \in S_{\tau}$ is the following subset of $\sigma$ :

$$
\sigma(A, B):=\left\{X \in \sigma \mid d_{\tau}(A, X)+d_{\tau}(X, B)=d_{\tau}(A, B)\right\}
$$

These are however only examples. In what follows, the whole usual system of definitions and theorems of Euclidean geometry are supposed to be known.

Now we are going to define the standard Cartesian coordinates in $S_{\tau}$. First we need a 3-frame.

Definition (A6) A 3-frame in $S_{\tau}$ consists of three pairwise orthogonal lines $\sigma_{x}, \sigma_{y}$, $\sigma_{z}$ in $S_{\tau}$, such that $\sigma_{x} \cap \sigma_{y} \cap \sigma_{z}=\{O\}$ and three events $X, Y, Z \neq O$ such that $X \in \sigma_{x}$, $Y \in \sigma_{y}$ and $Z \in \sigma_{z} . O$ is called the origin of the frame (Fig. 7). Let us introduce the following notations:

$$
\begin{aligned}
\sigma_{x}^{+}:= & \left\{P \in \sigma_{x} \mid \operatorname{Bet}_{\tau}(X, O, P)\right\} \\
\sigma_{x}^{-}:= & \left(\sigma_{x} \backslash \sigma_{x}^{+}\right) \cup\{O\} \\
\sigma_{y}^{+}:= & \left\{P \in \sigma_{y} \mid \operatorname{Bet}_{\tau}(Y, O, P)\right\} \\
\sigma_{y}^{-}:= & \left(\sigma_{y} \backslash \sigma_{y}^{+}\right) \cup\{O\} \\
\sigma_{z}^{+}:= & \left\{P \in \sigma_{z} \mid \operatorname{Bet}_{\tau}(Z, O, P)\right\} \\
\sigma_{z}^{-}:= & \left(\sigma_{z} \backslash \sigma_{z}^{+}\right) \cup\{O\}
\end{aligned}
$$

The origin of the 3 -frame is arbitrary, although it is a natural choice to take the " $\tau$ event" of the standard clock as origin. 
In the following definition we give the operational definition of space tags in one given $S_{\tau}$. Let us call them $\tau$-space tags.

Definition (A7) Let $A$ be an arbitrary event in $S_{\tau}$. Take a line segment $\sigma(B, C) \ni A$ parallel to $\sigma_{z}$ (Fig. 7). Take another line segment $\sigma(A, D)$ orthogonal to $\sigma_{z}$ such that $D \in \sigma_{z}$. Let $\sigma(O, E)$ be a line segment parallel to $\sigma(A, D)$ such that $E \in \sigma(B, C)$. Finally, take the line segments $\sigma(E, F)$ and $\sigma(E, G)$ such that $\sigma(E, F)$ is parallel to $\sigma_{x}$ and $F \in \sigma_{y}$, and $\sigma(E, G)$ is parallel to $\sigma_{y}$ and $G \in \sigma_{x}$. Now, the $\tau$-space tags are defined as follows:

$$
\begin{aligned}
& x_{\tau}(A):=\left\{\begin{array}{rrr}
d_{\tau}(G, O) & \text { if } & G \in \sigma_{x}^{+} \\
-d_{\tau}(G, O) & \text { if } & G \in \sigma_{x}^{-}
\end{array}\right. \\
& y_{\tau}(A):=\left\{\begin{array}{rrr}
d_{\tau}(F, O) & \text { if } & F \in \sigma_{y}^{+} \\
-d_{\tau}(F, O) & \text { if } & F \in \sigma_{y}^{-}
\end{array}\right. \\
& z_{\tau}(A):=\left\{\begin{array}{rrr}
d_{\tau}(D, O) & \text { if } & D \in \sigma_{z}^{+} \\
-d_{\tau}(D, O) & \text { if } & D \in \sigma_{z}^{-}
\end{array}\right.
\end{aligned}
$$

It must be emphasized that with the above definitions we only defined the space tags in a given set of simultaneous events $S_{\tau}$. Yet, we have no connection whatsoever between two $S_{\tau}$ and $S_{\tau^{\prime}}$ if $\tau \neq \tau^{\prime}$. In principle, there exist "infinitely" many possible bijections between the different $S_{\tau}$ 's. This is true, even if we prescribe that the bijection must be an isomorphism preserving distances.

Intuitively, a time sequence $\gamma(\tau)$ satisfying that

$$
\begin{aligned}
& x_{\tau}(\gamma(\tau))=\text { const. } \\
& y_{\tau}(\gamma(\tau))=\text { const. } \\
& z_{\tau}(\gamma(\tau))=\text { const. }
\end{aligned}
$$

corresponds to a localized physical object being at rest. "At rest"-relative to what? The actual behavior described by these equations depends on how the different 3frames are chosen in the different $S_{\tau}$ 's. One might think that an object is at rest if equations (11)-(13) hold in one and the same 3 -frame in all $S_{\tau}$. But, what does it mean that "one and the same 3 -frame in all $S_{\tau}$ "? When can we say that a line segment $\sigma_{x}^{\prime}$ in $S_{\tau^{\prime}}$ is the same 3-frame axis as $\sigma_{x}$ in $S_{\tau}$ ? When can we say that an event $A^{\prime}$ is in the same place in $S_{\tau^{\prime}}$ as event $A$ in $S_{\tau}$ ?

When we are seeking for a correspondence between $S_{\tau}$ and $S_{\tau^{\prime}}$, our aim is not simply to find a mathematically "canonical" bijection-whatever it means. What we wish is a one-to-one map

$$
\mathbb{T}_{\tau}^{\tau^{\prime}}: S_{\tau} \rightarrow S_{\tau^{\prime}}
$$

of natural physical meaning:

(a) It must be defined by means of physical operations. 
(b) For all $A, B \in S_{\tau}$, we require that $d_{\tau^{\prime}}\left(\mathbb{T}_{\tau}^{\tau^{\prime}}(A), \mathbb{T}_{\tau}^{\tau^{\prime}}(B)\right)=d_{\tau}(A, B)$.

(c) It must reflect our intuition about being "at rest". (For example, in our traditional language, if the standard clock moves along a time-like straight line of the Minkowski space-time, $\mathbb{T}_{\tau}^{\tau^{\prime}}$ must be equal to the map $(\tau, x, y, z) \mapsto\left(\tau^{\prime}, x, y, z\right)$, in the frame of reference of the standard clock. Of course, this example should be understood only intuitively.)

We have already defined a concept of the unique rest time sequence through every event. So, condition (c) basically means that for any rest time sequence $\gamma$ we require that $\mathbb{T}_{\tau}^{\tau^{\prime}}(\gamma(\tau))=\gamma\left(\tau^{\prime}\right)$. In fact, we will base the connection between different time slices on the rest time sequences:

\section{Definition (A8)}

$$
\begin{aligned}
\mathbb{T}_{\tau}^{\tau^{\prime}}: S_{\tau} & \rightarrow S_{\tau^{\prime}} \\
A & \mapsto \mathbb{T}_{\tau}^{\tau^{\prime}}(A)=\gamma\left(\tau^{\prime}\right)
\end{aligned}
$$

where $\gamma$ is a rest time sequence such that $A=\gamma(\tau)$. Let us call $\mathbb{T}_{\tau}^{\tau^{\prime}}$ the time shift between $S_{\tau}$ and $S_{\tau^{\prime}}$. It follows from (E1) and Lemma 1 that this definition is sound and $\mathbb{T}_{\tau}^{\tau^{\prime}}$ is a distance preserving bijection. Now we have everything at hand to define the space tags of events:

Definition (A9) Let $A$ be an arbitrary event. The absolute space tags of $A$ are defined as follows:

$$
\begin{aligned}
& \xi_{1}(A):=x_{0}\left(\mathbb{T}_{\tau(A)}^{0}(A)\right) \\
& \xi_{2}(A):=y_{0}\left(\mathbb{T}_{\tau(A)}^{0}(A)\right) \\
& \xi_{3}(A):=z_{0}\left(\mathbb{T}_{\tau(A)}^{0}(A)\right)
\end{aligned}
$$

Thus, we are given the absolute space and time tags for every event: $\xi_{1}(A), \xi_{2}(A)$, $\xi_{3}(A), \tau(A)$.

\section{Inertial motion}

A remark is in order on the empirical facts (E1)-(E13) to which we refer in constructing the space and time tags. When I call them empirical facts I mean that they ought to be true according to our ordinary physical theories. The ordinary physical theories are however based on the ordinary, problematic, space and time conceptions, relaying on "reference frames realized by rigid bodies" and the likes, without proper, non-circular, empirical definitions. Thus, especially in the context of defining the two most fundamental physical quantities, distance and time, we must not regard our ordinary physical 
theories as empirically meaningful and empirically confirmed claims about the world. Whether these statements are true or not is, therefore, an empirical question, and it is far from obvious whether they would be completely confirmed if the corresponding experiments were performed with higher precision, similar to the recent GPS measurements, especially for larger distances. Strangely enough, according to my knowledge, these very fundamental facts have never been tested experimentally; no textbook or monograph on space-time physics refers to such experimental results.

So, the best we can do is to believe that our physical theories based on the usual sloppy formulation of spatio-temporal concepts are true (in some sense) and to consider the predictions of these theories as empirical facts. However, as the following analysis reveals, it is far from obvious whether the predictions of the believed theories really imply (E1)-(E13).

Throughout the definition of space and time tags, we avoided the term "inertial", and because of a good reason. First of all, if "inertial" is regarded as a kinematical notion based on the concept of straight line and constancy of velocity, then it cannot be antecedent to the concept of space-time tags. If, on the other hand, it is understood as a manner of existence of a physical object in the universe, when the object is undergoing a free floating, in other words, when it is "free from forces", then the concept is even more problematic. The reason is that "force" is a concept defined through the deviation from the trajectory of inertial motion (first circularity), and neither the inertial trajectory nor the measure of deviation from it can be expressed without spatio-temporal concepts; consequently, they cannot be antecedent to the definition of space and time tags (second circularity). So there is no precise, non-circular definition of inertial motion. It is to be emphasized that this operational/logical circularity is a problem even in a special relativistic/flat/local space-time; and, therefore, it has nothing to do with the problem of conventionality of demarcation between "inertial" or "geodetic" motion versus gravitation as universal force (cf. Märzke and Wheeler's 1964).

According to our believed special relativistic physical theory, space-time is a 4dimensional Minkowski space and inertial trajectory is a time-like straight line in the Minkowski space. Since we are prior to the empirical definitions of the basic spatiotemporal quantities, we cannot regard this claim as an empirically confirmed physical theory. Nevertheless, let us assume for a moment that our special relativistic theory is the true description of the world "from God's point of view". It is straightforward to check that all the facts (E1)-(E13) are true if 1) the standard clock moves along an inertial world line in the Minkowski space-time and 2) it reads the proper time, that is, it measures the length of its own word line, according to the Minkowski metric. However, we human beings can know neither whether the standard clock (chosen by us) is of inertial motion in God's Minkowskian space-time nor whether it reads the proper time. What if these conditions fail? What does special relativistic kinematics say about (E1)-(E13) if the standard clock is accelerated and/or it does not read the proper time?

In order to answer this question, we have to follow up the operational definitions (A1), (A2),... and calculate whether statements (E1), (E2),.. are true or not if the standard clock moves along a given world line $\gamma$ and the "time" it reads is, say, a given function of the Minkowskian coordinate time, $\chi(t)$. Although the task is straightfor- 
ward, the calculation is too complex to give a general answer in details. Fortunately, we do not need all the details: the essential fact is that if we really can go through the whole operational procedure, and (E1)-(E13) are true, then, at the end, we obtain a coordination of events such that the equation describing the trajectory of a signal in the space of the four coordinates is

$$
\left(\xi_{1}(\tau)-\xi_{10}\right)^{2}+\left(\xi_{2}(\tau)-\xi_{20}\right)^{2}+\left(\xi_{3}(\tau)-\xi_{30}\right)^{2}=c^{2} \tau^{2}
$$

Now, due to the Alexandrov-Zeeman theorem (Alexandrov 1950; Zeeman 1964), one can derive the following results.

Theorem Facts (E1)-(E13) are true if and only if the standard clock moves along an inertial world line and reads a time $\chi(t)$ which is a linear function of the Minkowskian coordinate time.

Due to this theorem, in accord with our intuition based on the believed physical theories, we can give an objective meaning to "inertial motion" by means of correctneither logically nor operationally circular-experiments: the standard clock is of inertial motion if statements (E1)-(E13) are true. Assuming that the standard clock is inertial, one can extend the concept for an arbitrary time sequence $\gamma(\tau)$ of events: $\gamma(\tau)$ corresponds to an inertial motion if the absolute space tags $\xi_{1}(\gamma(\tau)), \xi_{2}(\gamma(\tau)), \xi_{3}(\gamma(\tau))$ are linear functions of the absolute time tag $\tau$.

There is a trivial but very important corollary of the above theorem: Imagine that we successfully perform two different coordinations of events by means of two different standard clocks. The theorem implies that the two coordinations are identical up to an almost Lorentz transformation.

Of course, the Alexandrov-Zeeman theorem applies if all of (E1)-(E13) are satisfied. It is perhaps interesting, that the essential condition is (E1). From an analysis by computer one finds the following result:

Result 1 There are no unique rest time sequences if the standard clock moves noninertially in a Minkowski space.

Still, one must emphasize, whether (E1)-(E13) are true or false is an open empirical question. Imagine that the standard clock is not inertial; for example (E1) is not satisfied. It would also mean that the clock chosen by us would be inappropriate for the definition of space-time tags. More exactly, we should have to stop at definition (A1). We could define the time tags but could not define the spatial notions, in particular the distances between simultaneous evens. Consequently, it is meaningless to talk about "non-inertial reference frame", "space-time coordinates (tags) defined/measured by an accelerated observer", and the likes. In the light of these consequences, it is an intriguing question whether the standard clock contemporary physical laboratories use for the coordination of physical events satisfies conditions (E1)-(E13), in particular (E1). 


\section{Absolute, relative, conventional}

I call $\tau(A)$ "absolute time" not in the sense of what Newton called "absolute, true and mathematical time", that is independent of any empirical definition, but in the sense of what the 20th century physics calls absolute time; it is "independent of the position and the condition of motion of the system of co-ordinates" (Einstein 1920, p. 51). The space and time tags $\xi_{1}(A), \xi_{2}(A), \xi_{3}(A), \tau(A)$ are absolute in the sense that they are not relative to a reference frame but prior to any reference frame. (The concept of "reference frame" is still not defined, and actually we do not need it.)

Absolute space and time tags are, of course, "relative" to the trivial semantical convention by which we define the meaning of the terms. They are "relative" to the etalon clock-like process we have chosen in the universe; and to the particular way in which the space and time tags are defined, including the usage of radio signals, the choice of " $\varepsilon=\frac{1}{2}$ ", etc. This kind of "relativism" is however common to all physical quantities having empirical meaning.

But there are two things that do not follow from this kind of conventionality. On the one hand, it does not follow that these physical quantities cannot describe objective features of physical reality; in spite of the obvious fact that these conventions play a constitutive role in the conceptual representation of the world. On the other hand, it does not follow either that there are no objective constraints on the semantical conventions themselves. In this last passage, I would like to give an example of how these objective constraints can restrict the semantical conventions defining absolute space and time tags.

There has been a long discussion in the literature about the conventionality of simultaneity. As it is obvious from (2), we chose the standard " $\varepsilon=\frac{1}{2}$-synchronization". This choice was a part of the trivial semantical convention defining the term "absolute time tag". It is, therefore, prior to any claim about the one-way or even round-trip speed of electromagnetic signals, because there is no such a concept as "speed" prior to the definition of time and space tags; it is, of course, prior to "the metric of Minkowski space-time", in particular to the "light-cone structure of the Minkowski space-time", because we have no words to tell this structure prior to the space and time tags; and it is prior to the causal order of physical events, because-even if we could know this causal order prior to temporality - we cannot know in advance how causal order is related with temporal order (which we have defined here). It is actually prior to any discourse about two locuses in space, because there is no "space" $\left(S_{\tau}\right)$ prior to definition (A1) and there is no concept of a "persistent space locus" prior to definitions (A3) and (A8).

So far, it seems, we are entirely free in the choice of the value of $\varepsilon$, that is in the choice of which objective feature of the physical reality- time $_{\varepsilon}$-we want to deal with. One might think that starting with some $\varepsilon$, that is with some time $e_{\varepsilon}$ tags $\tau_{\varepsilon}(A)$ and the corresponding $\varepsilon$-simultaneity slices $S_{\tau}^{\varepsilon}$, one finally obtains some space $\varepsilon_{\varepsilon}$ tags $\xi_{1}^{\varepsilon}(A), \xi_{2}^{\varepsilon}(A)$, and $\xi_{3}^{\varepsilon}(A)$, corresponding to the given value of $\varepsilon$. This is true only if we can go through all the operational definitions (A1)-(A13), and all the empirical facts (E1)-(E13) are true for the given $\varepsilon \neq \frac{1}{2}$.

This is, however, not necessarily the case. For, imagine we repeat the operations described in (A1), (A2) and (A3) with some $\varepsilon \neq \frac{1}{2}$, and obtain the concept of a (rest 
time sequence $)_{\varepsilon}$. Then, we encounter the question of whether the crucial empirical fact (E1) is true or not. Normally, in case of $\varepsilon=\frac{1}{2}$, we assumed that there exists a unique rest time sequence through every event. This assumption was confirmed by Result 1 derived from our believed physical theories. But, a similar computer calculation in case of $\varepsilon \neq \frac{1}{2}$ leads to the following result:

Result 2 Fact $(E 1)$ is never true if $\varepsilon \neq \frac{1}{2}$, no matter if the standard clock moves along an inertial world line, and no matter if the clock reads the proper time along its world line.

Again, whether or not (E1) is true is an open empirical question in both the $\varepsilon=\frac{1}{2}$ and the $\varepsilon \neq \frac{1}{2}$ cases. Nevertheless, assuming that the future empirical findings will confirm what our present physical theories tell about (E1), there seems no way to build up the spatial concepts ( rest $_{\varepsilon}$, distance ${ }_{\varepsilon}$, space $_{\varepsilon}$ tags, etc.) operationally, if $\varepsilon \neq \frac{1}{2}$. And, given that our aim is to define not only the temporal but also the spatial concepts, this is a strong experimentally testable argument against the $\varepsilon \neq \frac{1}{2}$-synchronization.

Acknowledgment The research was partly supported by the OTKA Foundation, No. K 68043.

\section{References}

Alexandrov, A. D. 1950. On Lorentz transformations. Uspekhi Math. Nauk 5: 187. (in Russian)

Brown, H. R. 2005. Physical Relativity. Space-time structure from a dynamical perspective. Oxford: Clarendon Press.

Butterfield, J. 2005. On the Persistence of Particles. Foundations of Physics 35: 233.

Einstein, A. 1920. Relativity: The Special and General Theory. New York: H. Holt and Company. Märzke, R. F. and Wheeler, J. A. 1964. Gravitation as geometry I:

the geometry of spacetime and the geometrodynamical standard meter, in Gravitation and relativity, H. Y. Chiu and W. F. Hoffmann (eds.), New York-Amsterdam: W. A. Benjamin Company.

Price, H. 1996. Time's arrow and Archimedes' point: new directions for the physics of time. New York, Oxford: Oxford Universoty Press.

Tarski, A. and S. Givant. 1999. Tarski's system of geometry. Bulletin of Symbolic Logic 5: 175 .

Zeeman, E. C. 1964. Causality implies the Lorentz group. Journal of Mathematical Physics 5: 490. 